\title{
Evaluación ambiental asociada a los vertimientos de aguas residuales generados por una empresa de curtiembres en la cuenca del río Aburrá
}

\section{Environmental assessment related to the sewage water discharge of a tannery company into Aburra river}

\author{
Diana Marcela Cuesta-Parra ${ }^{1}$, Carol Lorena Velazco-Rincón ${ }^{2}$, Julián Camilo Castro-Pardo ${ }^{3}$
}

\begin{abstract}
${ }^{1}$ Grupo de procesos de separación no convencional, Programa de Ingeniería Química, Fundación Universidad de América, Colombia. Orcid: 0000-0001-9287-2452.Email: diana.cuesta@profesores.uamerica.edu.co

${ }^{2}$ Grupo de procesos de separación no convencional, Programa de Ingeniería Química, Fundación Universidad de América, Colombia. Orcid: 0000-0001-6155-2894.Email: carol.velazco@estudiantes.uamerica.edu.co

${ }^{3}$ Grupo de procesos de separación no convencional, Programa de Ingeniería Química, Fundación Universidad de América, Colombia. Orcid: 0000-0002-6190-1748. Email: julian.castro@estudiantes.uamerica.edu.co
\end{abstract}

Recibido: noviembre 10, 2017. Aceptado: enero 27, 2018. Versión final: marzo 30, 2018.

\begin{abstract}
Resumen
Las industrias de curtiembre utilizan sustancias químicas orgánicas e inorgánicas para realizar sus operaciones, lo que genera vertimientos contaminantes que son descargados a las fuentes superficiales. En la presente investigación se evaluó el impacto ambiental asociado a los vertimientos de una empresa de curtiembres ubicada en el municipio de Copacabana, Antioquia, caracterizando el área de influencia directa e indirecta afectada por las descargas de la empresa al río Aburrá. Se aplicó el método Conesa (basado en el método de la matriz causa-efecto e involucrando la matriz de Leopold y el método Instituto Batelle-Columbus). Los resultados lograron identificar como impacto significativo el uso y la alteración fisicoquímica del recurso hídrico, especialmente en las etapas del proceso de la curtiembre, relacionados con el remojo, el pelambre, el desencalado, la purga, el piquelado, el curtido, el recurtido y el teñido. Con el fin de verificar la afectación de la descarga de los vertimientos de la empresa sobre la fuente hídrica, fueron simulados los parámetros de $\mathrm{pH}$, la conductividad, la DBO (rápida y lenta), el caudal y la temperatura, usando el modelo QUAL2K de la EPA. En este estudio se determinó que la DBO rápida vertida aumenta la concentración de $167 \mathrm{mg} \mathrm{O} / \mathrm{L}$ hasta $185 \mathrm{mg} \mathrm{O}_{2} / \mathrm{L}$ en el tramo estudiado, se aleja significativamente del objetivo de calidad del río Aburrá de $50 \mathrm{mg} \mathrm{O} / 2 / \mathrm{L}$ y supera los límites permisibles relacionados en la Resolución 631 del 2015 en los parámetros DQO y $\mathrm{DBO}_{5}$, en un $203 \%$ y $150 \%$. Los parámetros de conductividad y temperatura no mostraron afectación en la fuente.
\end{abstract}

Palabras clave: afectación; carga; cuenca; impacto; materia orgánica; modelación.

\begin{abstract}
Tannery industries use organic and inorganic chemicals to perform their operations by generating pollutant discharges that are discharged to surface sources. This study evaluated the environmental impact associated with the dumping of a tannery company located in the Municipality of Copacabana, Antioquia, characterizing the area of direct and indirect influence affected by the company's discharges in to river Aburrá. The Conesa method was applied (based on the cause-effect matrix method involving the Leopold matrix and the Batelle-Columbus Institute method). According to the results, it was possible to identify as a significant impact as a significant impact the use and physicochemical alteration of water resources, especially in the tanning process related to soaking, hair removal, dewatering, purging, ISSN impreso: 1657-4583, ISSN en línea: 2145 - 8456

Este artículo puede compartirse bajo la licencia CC BY-ND 4.0 y se referencia usando el siguiente formato: D. Cuesta-Parra, C. Velazco-Rincón, J. Castro-Pardo, "Evaluación ambiental asociada a los vertimientos de aguas residuales generados por una empresa de curtiembres en la cuenca del río Aburrá,” Rev. UIS Ing., vol. 17, no. 2, pp. 141-152, 2018. Doi: https://doi.org/10.18273/revuin.v17n2-2018013
\end{abstract}


pickling, tanning, retanning and dyeing.The parameters of $\mathrm{pH}$, Conductivity, $\mathrm{BOD}$ (fast and slow), flow rate and temperature using the QUAL2K model of the EPA were simulated in order to verify the impact of the discharge of the company's spills on the water source.In this study, it was determined that the rapid BOD discharged increases the concentration of $167 \mathrm{mg} \mathrm{O}_{2} / \mathrm{L}$ up to $185 \mathrm{mg} \mathrm{O}_{2} / \mathrm{L}$ in the studied section, moving significantly away from the quality objective of the Aburra river of $50 \mathrm{mg} \mathrm{O} / 2$ and exceeding the limits permissible in Resolution 631 of 2015 in the parameters $\mathrm{COD}$ and $\mathrm{BOD}_{5}$, by $203 \%$ and $150 \%$. The parameters of Conductivity and temperature did not show affectation in the source.

Keywords: affectation; impact; load; modeling; organic matter; watershed.

\section{Introducción}

El curtido de pieles es una actividad que se realiza con el fin de mejorar las propiedades de las pieles provenientes de especies animales. Para su tratamiento se usan sustancias químicas nocivas como hidrocarburos aromáticos policíclicos, sustancias halogenadas, metales pesados, taninos, azufre, entre otras, esto produce residuos tóxicos y materia orgánica como pelos y sangre. Esta industria se distingue por verter a las fuentes superficiales las cargas significativas de DBO y DQO, lo que reduce el oxígeno disuelto presente en estos efluentes.

En Colombia la actividad inició en los años 20, las curtiembres en Colombia están sectorizadas en Nariño (64), Quindío (27), Risaralda (1), Cundinamarca (190), Antioquia (7), Atlántico (2), Valle del Cauca (22), Bogotá (350), Tolima (8), Bolívar (1), Santander (4) y Huila (1) [1]. La afectación por parte de esta industria la reciben los ríos Pasto, Tunjuelo y Aburrá, entre otros.

De acuerdo con el artículo 42 del Decreto 3930 de 2010 emitido por el Ministerio de Ambiente y Desarrollo Sostenible, uno de los requisitos para solicitar el permiso de vertimientos a una fuente superficial es la Evaluación Ambiental del Vertimiento. La metodología aplicada permitió la valoración de la afectación producida por los vertimientos de una empresa de curtiembres al río Aburrá, en las inmediaciones del municipio de Copacabana, la cual incluye una evaluación de orden cualitativo y cuantitativo mediante el método de Conesa [2]. En el análisis de los resultados se realizó la comparación con los límites permisibles para el vertimiento incluidos en la legislación local y nacional. La modelación de calidad del agua en la fuente receptora se realizó con el Programa QUAL2K de la Agencia de Protección Ambiental de Estados Unidos, con el fin de predecir el comportamiento de la carga orgánica proveniente en el vertimiento y su afectación sobre el recurso hídrico.

\section{Marco teórico}

El curtido de pieles se realiza desde la antigüedad, empleando sustancias para curtir, como grasas animales y minerales, humo, productos vegetales ricos en taninos, cereales, minerales, mezclas de estos, productos químicos, entre otros [3], para la transformación en cuero de las pieles de los animales [4] bovinos, ovinos, porcinos, caprinos y reptiles [5]. Estos materiales son empleados en prendas de vestir, muebles o calzado. Su transformación requiere una serie de etapas llevadas a cabo en las curtiembres: ribera, proceso de curtido, poscurtido y acabado.

La primera etapa es la ribera, esta se realiza en la curtiembre, después del descarne realizado en las centrales de sacrificio, el acondicionamiento en los proveedores, el traslado, el tiempo de almacenamiento, los tratamientos de conservación y el estado de la piel. En la ribera se realiza la limpieza y la preparación de la piel, para esto se remueve el pelo o la lana y la endodermis, según el animal del que proviene la materia prima, la piel es hidratada y se remueven impurezas [6]. La ribera está compuesta por varias subetapas, como el remojo, el pelambre, el desencalado, la purga enzimática y el lavado; en esta se usan sustancias como sulfuro de sodio, hidróxido de calcio, tensoactivos, sulfato de amonio, bisulfito de sodio, que quedan mezcladas en los residuos líquidos, así como el pelo, partes de la epidermis, fibras de colágeno, sangre y grasa [7].

El curtido es el proceso que permite la transformación de la piel en cuero. En esta se estabiliza el colágeno, usando agentes curtientes provenientes de vegetales o sales de cromo trivalente. En el curtido con sales de cromo se realizan cuatro subetapas: piquelado, curtido, basificado y neutralización. Durante el piquelado se prepara el cuero eliminando la cal combinada con el colágeno y se interrumpen completamente las reacciones de las enzimas adicionando ácidos y sales. Posteriormente la piel ingresa al curtido donde se emplean sales inorgánicas de cromo. Este proceso permite que la estructura adquiera estabilidad. Para que el cromo trivalente penetre en la piel, esta es sumergida en solución, con lo que se logra que los componentes orgánicos e inorgánicos 
reaccionen; en esta operación se aumenta la temperatura. La acción del agente curtiente es facilitada por la adición de sales alcalinas (bicarbonato de sodio, óxido de magnesio, carbonato de sodio, entre otras) que aumentan el pH de la solución y favorecen la reacción con los ligantes orgánicos durante el basificado, para finalizar se lleva el cuero a un proceso de neutralización aumentando el pH para eliminar la acidez. En la etapa de poscurtido o recurtido se repite el proceso anterior, con el fin de mejorar la resistencia, la manejabilidad y la suavidad, para esto se complementa con procedimientos como teñido, engrase, escurrido y estirado, según sea necesario. Para el caso del terminado húmedo, cuando el cuero es curtido al cromo se requiere neutralizar para elevar el $\mathrm{pH}$ inicial, si el producto lo requiere se blanquea y se tiñe o tintura con colorantes directos que pueden ser ácido o básicos [5].

En el acabado se busca principalmente realizar operaciones de superficie. A este proceso ingresan los cueros provenientes de recurtido y pasan por un secado que reduce la humedad del material (16-22 \%) [5], debido al procedimiento anterior, el cuero pierde flexibilidad, y de esta manera se requiere de un ablandamiento que permita obtener un producto con las características requeridas; posteriormente se somete el cuero al lijado para igualar y corregir defectos del lado de la flor. Esta actividad se conoce también con el nombre de esmerilado. Como es de esperarse, quedan residuos finos que deben ser removidos del cuero, y para ello se realiza un sacudido o desempolvado.

Para lograr remover la humedad y los solventes que estén impregnados en el cuero, se cuelgan y se evaporan los compuestos mencionados, lo que da lugar a una serie de procedimientos opcionales, como el esmerilado, el desempolvado y el pigmentado [8]; luego de estos pasos se prensa o plancha el cuero. Para proteger cada una de las modificaciones realizadas, es importante lacar el material, de manera que se logre un acabado de calidad, con las medidas requeridas para ser almacenado y comercializado.

Los vertimientos generados en la industria de curtido, al ser descargados de forma directa en los cuerpos receptores de agua, provocan efectos negativos en los usos posteriores de estos, lo que modifica la calidad del agua y afecta negativamente la vida acuática [9].

Las etapas convencionales de ribera y de curtido producen el $90 \%$ de la contaminación total de una curtiembre [10]. En la etapa de precurtido o ribera se producen variaciones significativas en el $\mathrm{pH}$, el aumento en la concentración de la DQO (Demanda Química de
Oxígeno), los STD (Sólidos Disueltos Totales), las sales como cloruros y sulfatos [11].

El proceso de pelambre representa el $84 \%$ de la carga de DBO (Demanda Bioquímica de Oxígeno), el $75 \%$ de la carga de DQO y el $92 \%$ de los SST (Sólidos Suspendidos Totales) de una curtiembre [12]. Durante la ribera se consumen grandes cantidades de agua en relación con el peso de las pieles, entre los desechos líquidos generados en una curtiembre. Aproximadamente el $65 \%$ [13] se producen en los procesos de esta etapa.

\section{Metodología}

La metodología aplicada para evaluación de impactos ambientales fue el método de Vicente Conesa [14]. Esta consiste en dos tipos de evaluaciones:

En la evaluación cualitativa, se identifican las acciones que pueden causar impactos sobre una serie de factores del medio, como la modificación del suelo, el cambio en las características fisicoquímicas del agua, la emisión de contaminantes, la generación y almacenamiento de residuos, la sobreexplotación de recursos, los cambios en el medio biótico y el deterioro del paisaje, relacionados con las actividades que se realizan en el proceso. El método requiere la aplicación de una matriz de doble entrada, en la que se cruzan los componentes del ambiente con las actividades del proyecto o del sistema de tratamiento [14].

La evaluación cuantitativa tiene como fin establecer a través de los factores ambientales considerados, los indicadores, la unidad de medida y la magnitud, para transformar estos valores en magnitudes representativas, no de su alteración, sino de su impacto neto sobre el ambiente. Los criterios de evaluación para determinar la importancia ambiental son la naturaleza, la extensión, la intensidad, el momento, la duración, la reversibilidad, la sinergia, la acumulación, el efecto, la periodicidad y la recuperabilidad. El resultado de la evaluación indica si un impacto es irrelevante, moderado, severo o crítico.

Para la modelación de las condiciones de afectación del río al recibir las descargas contaminantes del proceso de curtiembre, se utilizó el software QUAL2K desarrollado por la EPA (Agencia de Protección Ambiental de Estados Unidos). El modelo QUAL2K emplea el software Excel como interfase gráfica y de acceso de datos. El modelo QUAL2K emplea el software Excel como interfase gráfica y de acceso de datos. El libro de Excel contiene varias hojas electrónicas desde donde se suministran los datos de entrada del modelo y desde donde este se ejecuta. Cada una de estas hojas electrónicas tiene un nombre que identifica el tipo de información que 
contiene: información general, información de la frontera aguas arriba (cabecera), descripción de los tramos del río, condiciones climatológicas (temperatura del aire, temperatura del punto de rocío, velocidad del viento, nubosidad y sombra), los valores de las tasas de reacción o constantes de calibración del modelo, la información correspondiente a los tributarios y las captaciones puntuales y distribuidas y la información de las estaciones aguas abajo de la cabecera.

Los datos requeridos para la modelación aguas arriba y aguas abajo del punto de vertido fueron tomados de la información publicada en la red de monitoreo de la calidad del agua del río Aburrá. Se tuvieron en cuenta las estaciones de monitoreo más cercanas.

- Niquía (E21) coordenadas 6²0'17,73" N y $75^{\circ} 31^{\prime} 32,57^{\prime}$ E, aguas arriba ubicada en el municipio de Bello.

- Ancón Norte (E12) coordenadas 6²2'16,21”N N y $75^{\circ} 29^{\prime} 21,29^{\prime \prime}$ E, aguas abajo ubicada en el municipio de Copacabana [15].

Los resultados de la caracterización fisicoquímica del vertimiento en los parámetros son los siguientes: $\mathrm{pH}$ analizado por método SM-4500 H+ electrodo selectivo; DQO por reflujo cerrado SM-5220 D; DBO5 por Método Winkler; electrodo selectivo de O2 SM-5210 B SM4500 O G; grasas y aceites por extracción SoxhletGravimetría SM-5520 D: sólidos suspendidos totales por gravimetría SM-2540 D; cromo total espectrofotometría de absorción atómica SM 3030H -3111D, y sulfuros por yodometría SM-4500 S2-F, que fueron suministrados por la empresa, y el análisis de calidad del agua fue realizado por un laboratorio acreditado por el Ideam.

\section{Resultados}

\subsection{Evaluación de impacto ambiental}

Con el uso de la herramienta para la evaluación cualitativa se identificó que, en las etapas de remojo, pelambre, desencalado y purgado, piquelado, neutralizado, curtido, recurtido y teñido, el impacto común es el consumo de agua y modificación de la calidad del recurso.

En la evaluación cuantitativa cada impacto ambiental identificado en la matriz se valora según los criterios de evaluación descritos en la metodología, para el método de Conesa. El resultado de la evaluación cuantitativa se analiza por componente en relación con las actividades del proceso productivo:

Para el componente agua las actividades de remojo, pelambre, desencalado y purga, piquelado, curtido y recurtido y teñido, los impactos ambientales asociados con el consumo de agua y la modificación de la calidad fisicoquímica del recurso hídrico se valoraron como significativos, por lo cual son prioritarias las actividades de mitigación, prevención y compensación. Para el componente suelo, el impacto valorado como significativo es la modificación de las características fisicoquímicas del suelo, por la producción, el almacenamiento, el transporte y la disposición de lodos y residuos peligrosos que se producen en el proceso y durante el tratamiento de aguas residuales. Dentro de las actividades de mitigación, se deben caracterizar los lodos con el fin de determinar su peligrosidad, cumpliendo con la normativa legal al respecto. Para el aire se determinó que, en las actividades de remojo, pelambre, desencalado y purga se producen olores provenientes de la descomposición de la materia orgánica, proteínas, sangre en las pieles cruda, además del uso de sulfuro de sodio. Pese a que se genera ruido, se tendría que realizar un estudio para determinar si ese ruido supera los límites permisibles; actualmente no hay quejas de la comunidad por emisiones de olores o de ruido. El componente fue calificado como moderado.

\subsection{Modelación de la afectación por vertimientos al río Aburrá}

Para la modelación de la afectación por la descarga de desechos líquidos provenientes de una curtiembre al río Aburrá, se tomó información de las estaciones de monitoreo de calidad del agua del área metropolitana del Valle de Aburrá, la estación previa al punto de descarga es Niquía (E21), ubicada en el municipio de Bello. La estación posterior al punto de descarga es Ancón Norte (E12), ubicada en el municipio de Copacabana. La distancia de separación entre las estaciones es $6,3 \mathrm{~km}$ en el cauce del río; el punto de vertimiento de la empresa se encuentra ubicado a 2,5 km de la estación Ancón Norte. La información de calidad del agua del río Aburrá, relacionada en la tabla 2, corresponde a las fechas 19 de febrero de 2014 y 26 de febrero de 2014. 
Evaluación ambiental asociada a los vertimientos de aguas residuales generados por una empresa de curtiembres en la cuenca del río Aburrá

Tabla 1. Identificación de impactos ambientales.

\begin{tabular}{|c|c|}
\hline Actividad & Impactos ambientales cualitativos \\
\hline Remojo & $\begin{array}{l}\text { En la evaluación cualitativa se identificó consumo de agua, modificación a las condiciones fisicoquímicas del } \\
\text { recurso. En el proceso se genera ruido por los motores de los bombos y hay afectación a la salud por residuos } \\
\text { líquidos. La actividad produce activación de la economía. }\end{array}$ \\
\hline Pelambre & $\begin{array}{l}\text { En la actividad de pelambre se presenta consumo de agua, modificación a las condiciones fisicoquímicas del } \\
\text { recurso, producción de residuos peligrosos, como lodos con sustancias químicas, envases y contenedores. El } \\
\text { ruido se produce por los motores de los bombos y hay afectación a la salud por el tipo de sustancias a las que } \\
\text { se expone el personal. La actividad produce activación de la economía }\end{array}$ \\
\hline Descanardo & $\begin{array}{l}\text { En esta actividad se generan residuos sólidos, peligrosos y biológicos, como el colágeno y los residuos de la } \\
\text { separación de las capas de la piel (material orgánico). La presencia de material particulado es evidente en la } \\
\text { operación, al igual que los olores por el contenido de sulfuros en el proceso. Hay afectación a la salud por el } \\
\text { tipo de sustancias a las que se expone el personal. }\end{array}$ \\
\hline Dividido & En el dividido se generan residuos sólidos que son dispuestos como ordinarios. \\
\hline $\begin{array}{l}\text { Desencalado y } \\
\text { purga }\end{array}$ & $\begin{array}{l}\text { En el desencalado se consume agua y se usan ácidos orgánicos que producen residuos líquidos y peligrosos, } \\
\text { además de emisiones de nieblas ácidas, ruido y afectación a la salud del personal expuesto. }\end{array}$ \\
\hline Piquelado & $\begin{array}{l}\text { En el piquelado se utilizan sustancias químicas similares a las etapas anteriores, y el consumo de agua es } \\
\text { equivalente. Los impactos generados son la producción de residuos líquidos y peligrosos, emisiones } \\
\text { atmosféricas y ruido. }\end{array}$ \\
\hline Curtido & $\begin{array}{l}\text { En el curtido se usan sales de cromo, lo que genera transformación en la calidad del agua, residuos peligrosos, } \\
\text { emisiones de metales pesados, ruido y afectación secundaria a suelos y ecosistemas. }\end{array}$ \\
\hline Rebajado & $\begin{array}{l}\text { En esta actividad no se usa agua, hay consumo de energía eléctrica y producción de residuos sólidos, retazos } \\
\text { de la materia prima y virutas, que se entregan finalmente como residuos ordinarios }\end{array}$ \\
\hline Neutralizado & $\begin{array}{l}\text { En la actividad de neutralizado se usan sales de sodio, las cuales reducen la acidez del agua. En este caso } \\
\text { específico, los residuos líquidos contienen sólidos disueltos que aumentan la DQO, residuos peligrosos. Se } \\
\text { produce ruido en la operación y hay afectación indirecta a ecosistemas y suelo }\end{array}$ \\
\hline Recurtido & $\begin{array}{l}\text { En el recurtido se repiten las operaciones de curtido; se usan sales de cromo. Hay modificación en la calidad } \\
\text { del agua vertida, se producen residuos peligrosos, emisiones de metales pesados, ruido, afectación secundaria } \\
\text { a suelos y ecosistemas. }\end{array}$ \\
\hline $\begin{array}{l}\text { Teñido y } \\
\text { Engrasado }\end{array}$ & $\begin{array}{l}\text { En esta operación unitaria se aplican grasas y aceites vegetales, se producen residuos peligrosos y afectación } \\
\text { a la salud y al medio por contaminación física. Hay ruido producido por el compresor y consumo de energía } \\
\text { eléctrica }\end{array}$ \\
\hline Secado & $\begin{array}{l}\text { Durante el secado son emitidos al medio gases, olores y vapores procedentes del secado, consumo de energía } \\
\text { eléctrica }\end{array}$ \\
\hline $\begin{array}{cc}\text { Pintado } \\
\text { planchado }\end{array}$ & $\begin{array}{l}\text { En el uso de pigmentos y colorantes para los procesos de teñido, se generan emisiones atmosféricas, residuos } \\
\text { peligrosos, residuos sólidos de retazos y virutas de material terminado. }\end{array}$ \\
\hline
\end{tabular}

Fuente: elaboración propia. 
Tabla 2. Parámetros de modelación.

\begin{tabular}{|c|c|c|c|c|}
\hline \multicolumn{5}{|c|}{ Calidad del agua del río Aburrá } \\
\hline Tipo de muestra & Aguas arriba & Aguas abajo & Aguas arriba & Aguas abajo \\
\hline Fecha & $2 / 19 / 2014$ & $2 / 19 / 2014$ & $2 / 26 / 2014$ & $2 / 26 / 2014$ \\
\hline Fase & 4 & 4 & 4 & 4 \\
\hline Campaña & 19 & 19 & 20 & 20 \\
\hline Estación & Niquía & Ancón Norte & Niquía & Ancón Norte \\
\hline Código & E21 & E12 & E21 & E12 \\
\hline \multicolumn{5}{|l|}{ Parámetro } \\
\hline T Agua $\left({ }^{\circ} \mathrm{C}\right)$ & 22,05 & 21,91 & 22,55 & 22,09 \\
\hline $\mathrm{pH}(\mathrm{U}$ de $\mathrm{pH})$ & 7,60 & 7,45 & 7,56 & 7,49 \\
\hline Oxígeno disuelto $(\mathrm{mg} / \mathrm{L})$ & 0,76 & 0,63 & 1,02 & 0,35 \\
\hline Conductividad eléctrica $(\mu \mathrm{s} / \mathrm{cm})$ & 451,00 & 450,00 & 514,00 & 521,00 \\
\hline Potencial redox $(\mathrm{mV})$ & & $-70,00$ & & $-36,65$ \\
\hline Turbiedad (NTU) & & 322,92 & & 229,40 \\
\hline DBO5 (mg/L) & 168 & 144 & 154,00 & 147,00 \\
\hline DQO (mg/L) & 255 & 186 & 255,00 & 241,00 \\
\hline P-Total (mg P/L) & 2,900 & 2,67 & 4,17 & 3,50 \\
\hline NTK (mg N/L) & 21,400 & 19,20 & 21,60 & 20,90 \\
\hline $\mathrm{SST}(\mathrm{mg} / \mathrm{L})$ & 256,00 & 284,00 & 327.00 & 266,93 \\
\hline BMWP Col valor & 1 & 1 & 8 & 1 \\
\hline BMWP Col Calidad & Muy crítica & Muy crítica & Muy crítica & Muy crítica \\
\hline ICACOSU Valor & 0,29 & 0,27 & 0,25 & 0,27 \\
\hline ICACOSU Calidad & Mala & Mala & Muy Mala & Mala \\
\hline Coliormes Totales $(\mathrm{UFC} / 100 \mathrm{ml})$ & $5,90 \mathrm{E}+10$ & $9,50 \mathrm{E}+10$ & $5.40 \mathrm{E}+07$ & $8.30 \mathrm{E}+07$ \\
\hline $\mathrm{Q}(\mathrm{m} 3 / \mathrm{s})$ & $\mathrm{N} / \mathrm{D}$ & 17,65 & 17,53 & 18.87 \\
\hline $\begin{array}{c}\text { Clasificación } \\
\text { Caudal }\end{array}$ & Medio & Medio & Bajo & Bajo \\
\hline E - Coli (UFC/100 ml) & $5.40 \mathrm{E}+06$ & $5.70 \mathrm{E}+05$ & $5.00 \mathrm{E}+06$ & $4.00 \mathrm{E}+06$ \\
\hline
\end{tabular}

Fuente: elaboración propia.

El caudal promedio del río en la estación Ancón Norte fue de $21,23 \mathrm{~m}^{3} / \mathrm{s}$, y para la estación de Niquía se tomó el caudal medio de $18,98 \mathrm{~m} 3 / \mathrm{s}$.

La empresa cuenta con un sistema de tratamiento de agua residual industrial compuesta por un sistema de pretratamiento que incluye cribado fijo y grueso, trapa de grasas y homogenización, el sistema primario conformado por oxidación catalítica para las aguas provenientes de pelambre y el sistema de flotación y clarificación para la totalidad de los residuos líquidos producidos. En la modelación de la afectación de la calidad del agua del río Aburrá se tuvo en cuenta el punto de vertimiento de la empresa. En este el caudal promedio de descarga fue de 1,48 L/s, medido en la caracterización 
Evaluación ambiental asociada a los vertimientos de aguas residuales generados por una empresa de curtiembres en la cuenca del río Aburrá

de agua residual tratada por parte de los laboratorios acreditados por el Ideam.

Debido a que en la caracterización de la empresa fueron analizados los parámetros de interés sanitario según la normativa legal, se decide que los parámetros incluidos en la modelación serán cinco: la temperatura, la Demanda Bioquímica de Oxígeno, el pH, la Demanda Química de Oxígeno y la conductividad eléctrica, los resultados de los análisis de los parámetros mencionados en el agua residual tratada de la curtiembre están relacionados en la tabla 3 .

Tabla 3. Parámetros de salida del agua residual tratada proveniente de la industria

\begin{tabular}{|l|l|l|l|l|}
\hline \multicolumn{1}{|c|}{ Parámetro } & \multicolumn{1}{c|}{ Unidad } & \multicolumn{1}{c|}{ Conc. } & Res. 631 2015 & Res. 2016 2012 \\
\hline $\mathrm{pH}$ & Unidades $\mathrm{pH}$ & $6,88-8,66$ & $6-9$ & $>6.5<8.5$ \\
\hline $\mathrm{DBO5}$ & $\mathrm{mg} \mathrm{O} / \mathrm{L}$ & 1502 & 600 & $<80$ \\
\hline $\mathrm{DQO}$ & $\mathrm{mg} \mathrm{O} / \mathrm{L}$ & 3633 & 1200 & $<150$ \\
\hline Grasas y aceites & $\mathrm{mg} \mathrm{grasas} / \mathrm{L}$ & 53 & 60 & $<20$ \\
\hline Sólidos suspendidos totales & $\mathrm{mg} \mathrm{SST} \mathrm{/L}$ & 683 & 600 & $<300$ \\
\hline Cromo total & $\mathrm{mg} \mathrm{Cr} / \mathrm{L}$ & 9,94 & 1.5 & $\mathrm{NA}$ \\
\hline Sulfuros totales & $\mathrm{mg} \mathrm{S} / \mathrm{L}$ & 241 & 3 & NA \\
\hline Conductividad (promedio) & $\mu \mathrm{s} / \mathrm{cm}$ & 31.940 & $\mathrm{NA}$ & $>120$ \\
\hline
\end{tabular}

Los resultados obtenidos en la modelación fueron comparados con la legislación para el cumplimiento de objetivos de calidad en la cuenca y los límites permisibles para vertimientos en fuentes superficiales; es decir, se tendrán en cuenta la Resolución 2016 del año 2012, emitida por el área metropolitana del Valle de Aburrá y la Resolución 0631 del 2015 del Ministerio de Ambiente y Desarrollo Sostenible.

Para la simulación de la mezcla, se revisó el caudal en el tramo seleccionado, en la estación de Niquía. El caudal fue de $18,98 \mathrm{~m}^{3} / \mathrm{s}$, este se toma como dato del cauce aguas arriba de la zona de descarga de efluentes líquidos de la empresa. La simulación muestra una fluctuación del caudal. En este se puede identificar la distancia a la que se realiza el vertimiento de las aguas residuales industriales de la curtiembre. Ubicado a los 2,5 km de la estación de monitoreo, el caudal del río Aburrá aumenta progresivamente hasta alcanzar los $18,98147 \mathrm{~m}^{3} / \mathrm{s}$, como se muestra en la gráfica 1. El caudal en Ancón Norte es de $21.23 \mathrm{~m}^{3} / \mathrm{s}$, lo que demuestra que no es el único flujo que puede estar recibiendo el río. El vertimiento aporta el $0,0077 \%$ del caudal total del río aguas abajo de la descarga realizada por la empresa.

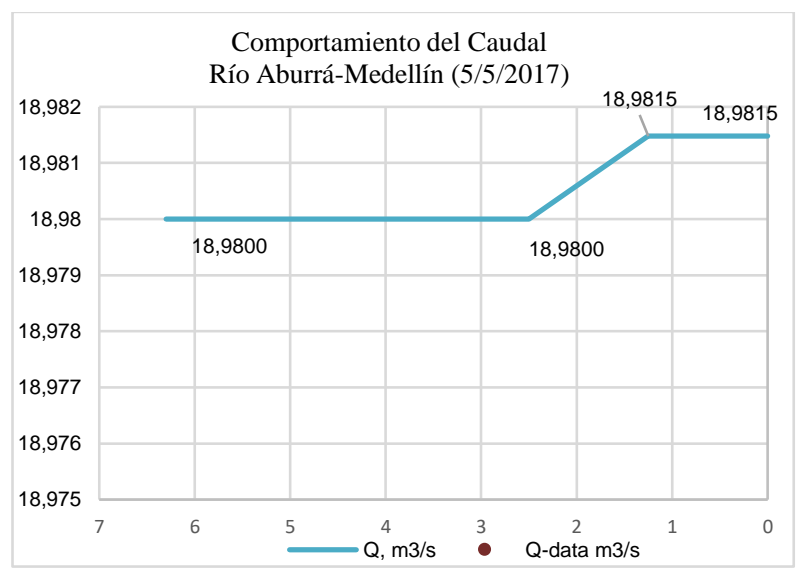

Gráfica 1. Caudal modelado para el río Aburrá (Medellín).

En algunos procesos industriales se requiere elevar la temperatura del agua. Si el agua residual es vertida en estas condiciones se producen efectos adversos a la vida acuática de los cuerpos de agua receptores, cambios en los coeficientes de transferencia de masa y en la concentración de oxígeno. La gráfica 2 representa la simulación realizada por el programa QUAL2K para la temperatura en el río Aburrá, específicamente en el tramo comprendido entre las estaciones de Niquía y de Ancón Norte. Según los reportes de las estaciones, la temperatura media del agua para el río es de $22,5^{\circ} \mathrm{C}$; el resultado reportado para el vertimiento fue una temperatura media de $25,2{ }^{\circ} \mathrm{C}$. 
En la simulación se demuestra que no hay afectación del vertimiento al afluente: por el contrario, aguas abajo acercándose al sitio de la estación de monitoreo de Ancón Norte existe una leve disminución de la temperatura del agua modelada para el cauce del río. El parámetro se encuentra entre el rango permitido, según el Área Metropolitana del Valle de Aburrá en sus informes de RedRío para una corriente de aguas superficiales cuyo rango de temperatura es de $18,5^{\circ} \mathrm{C}$ a $28^{\circ} \mathrm{C}[16]$.

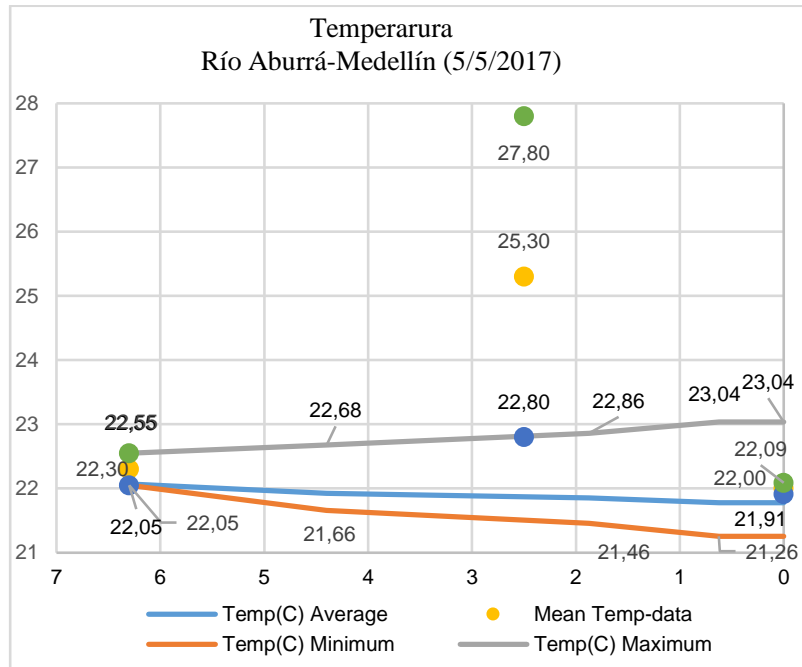

Gráfica 2. Temperatura modelada para el tramo Niquía-Ancón Norte.

La conductividad eléctrica es una variable que está directamente relacionada con la cantidad de sólidos disueltos totales. En la información de conductividad entregada por la empresa de $31.940 \mu \mathrm{s} / \mathrm{cm}$; sin embargo, el caudal que ingresa a la fuente superficial es inferior al caudal del río reportado en la estación de Niquía, por lo tanto, ingresa al volumen de control, lo que significa que posteriormente es diluido en el cauce, como se muestra en la gráfica 3. El objetivo de recuperación del río establece para este parámetro que el vertimiento debe tener una conductividad menor a $120 \mu \mathrm{s} / \mathrm{cm}$, pero actualmente el río no cumple con estas características. En la simulación se muestra como en la estación Ancón Norte no hay cambios significativos en la conductividad, los últimos kilómetros el valor se estabiliza en un valor de $456 \mu \mathrm{s} / \mathrm{cm}$.

La Demanda Bioquímica de Oxígeno es un parámetro que determina la carga contaminante que pueden generar los desechos de carácter biológico de la curtiembre, para el modelo se representa como el $\mathrm{DBO}_{5}$ correspondiente a la materia orgánica que se oxida o se degrada de forma rápida. En la gráfica 4 se observa que la descarga de la empresa es $1,502 \mathrm{mgO}_{2} / \mathrm{L}$. De acuerdo con los datos tomados de la estación de monitoreo la concentración de $\mathrm{DBO}_{5}$ aguas arriba al vertimiento es de $168 \mathrm{mgO}_{2} / \mathrm{L}$. Al ingresar los datos de la concentración de $\mathrm{DBO}_{5}$ del vertimiento, el modelo muestra un aumento significativo en el tramo pasando de $168 \mathrm{mgO}_{2} / \mathrm{L}$ en Niquía (estación aguas arriba) hasta aproximadamente $185 \mathrm{mgO}_{2} / \mathrm{L}$ en Ancón Norte (estación aguas abajo).

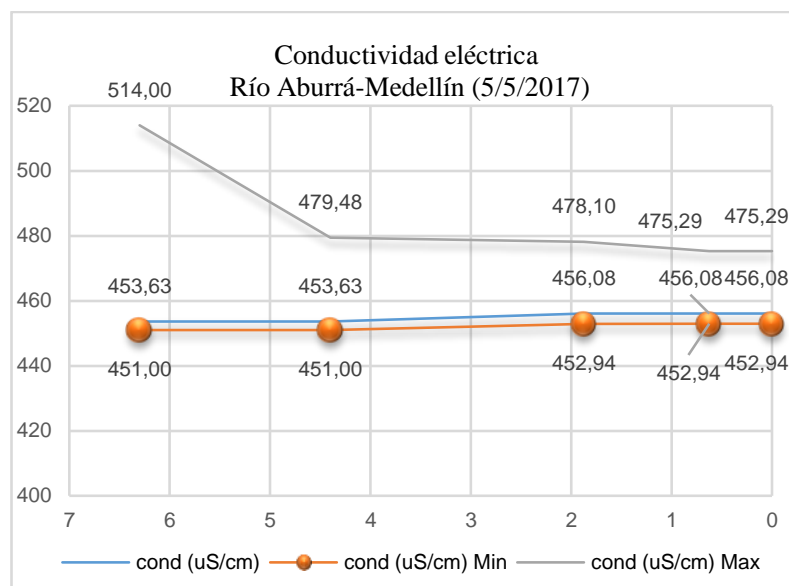

Gráfica 3. Conductividad eléctrica modelada en el río Aburrá (Medellín).

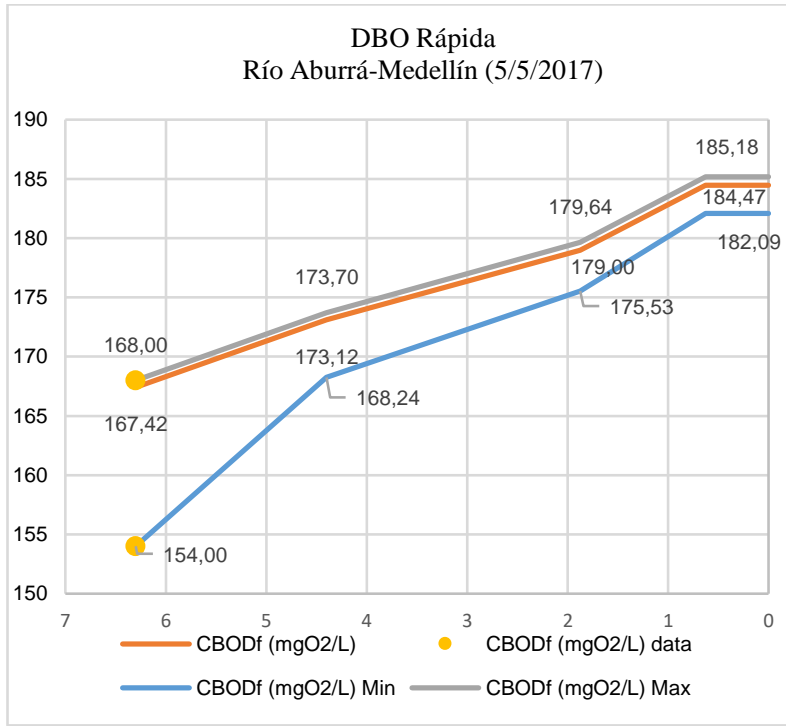

Gráfica 4. Ampliación $\mathrm{DBO}_{5}$ modelada por el software.

El modelo muestra que después del vertimiento la pendiente de este parámetro se vuelve más pronunciada. La grafica 4 muestra la afectación producto de la descarga de materia orgánica de la empresa al río, debido al aumento de la concentración, A mayor valor de DBO, mayor es la contaminación del agua [18]. Cabe resaltar que cuando el cauce del río se acerca a la estación de Ancón Norte aproximadamente a $0,7 \mathrm{~km}$ aguas arriba de 
Evaluación ambiental asociada a los vertimientos de aguas residuales generados por una empresa de curtiembres en la cuenca del río Aburrá

esta la $\mathrm{DBO}_{5}$ presenta un comportamiento constante de $185 \mathrm{mgO}_{2} / \mathrm{L}$, lo cual demuestra la estabilización de la carga en este punto. El parámetro $\mathrm{DBO}_{5}$ en el tramo de estudio está por encima del límite establecido por los objetivos de calidad de 5-10 años para este recurso hídrico, en la Resolución 2016 del Área Metropolitana del año 2012, debido a que su valor debe ser menor a 50 $\mathrm{mgO}_{2} / \mathrm{L}$.

La Demanda Química de Oxígeno se usa en el programa de modelación, para calcular la DBO lenta, la cual se obtiene restando la demanda química de oxígeno total menos la $\mathrm{DBO}_{5}$. (Díaz, 2004) Esta DBO refleja el carbono de descomposición lenta (sales, grasas y aceites).

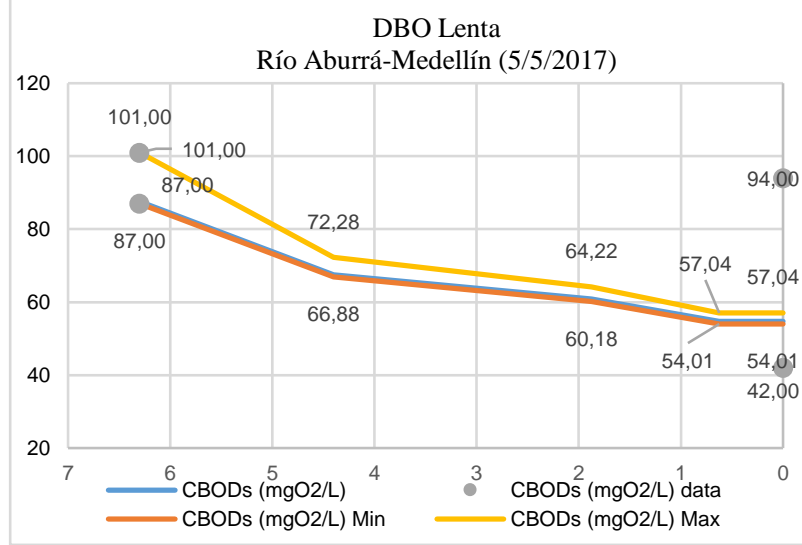

Gráfica 5. Ampliación DQO modelada por el modelo QUAL2K

Para el parámetro DQO, la gráfica 5 muestra que no hay afectación al tramo del cauce principal, teniendo en cuenta que este parámetro integra la DBO rápida y lenta, y se puede asociar con los resultados reportados en la gráfica 4, donde la concentración de materia orgánica oxidable aumenta la carga en el río, mientras que la DBO lenta probablemente procedente de sales inorgánicas oxidables se disuelven en el cauce. Al relacionar los resultados de la modelación de la demanda química de oxígeno con los objetivos de calidad para el tramo, se encuentran concentraciones superiores a $100 \mathrm{mgO}_{2} / \mathrm{L}$.

En la información de la concentración de oxígeno disuelto incluida en la modelación, los niveles más bajos de oxígeno disuelto del río se presentan en la gráfica 6 , a los 6,3 km entre las dos estaciones, es posible que la carga orgánica de la empresa reduzca el oxígeno a los $2,5 \mathrm{~km}$.

Las concentraciones inferiores a $4 \mathrm{mgO}_{2} / \mathrm{L}$ amenazan la vida acuática [17].

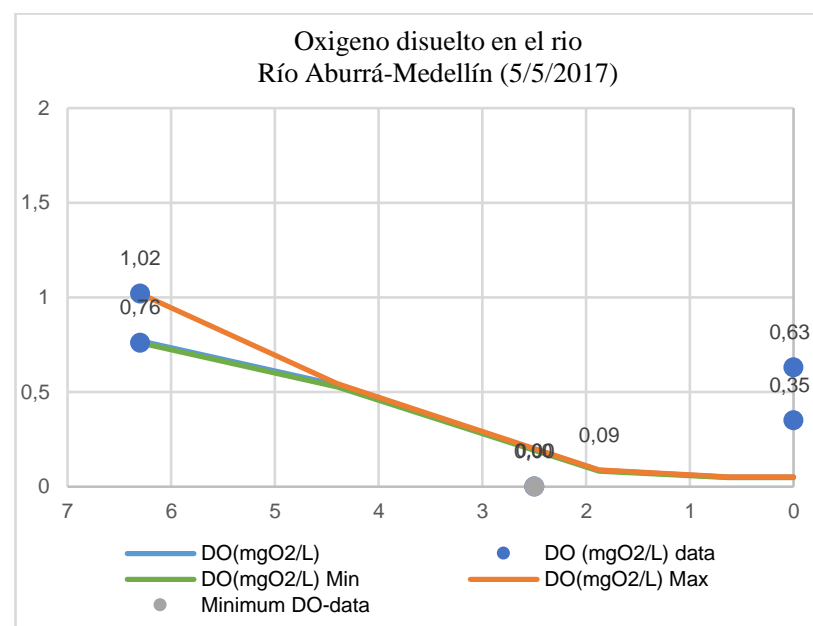

Gráfica 6. Oxígeno Disuelto modelado para el tramo NiquíaAncón Norte.

El pH es un parámetro químico indicador de la calidad del agua que muestra la cantidad de hidrogeniones $(\mathrm{H}+)$ que posee el agua y puede afectar la vida de animales y plantas acuáticas. El pH en la salida de la PTARI arrojó valores entre 6,88 y 8,66 unidades de $\mathrm{pH}$. El valor máximo está por fuera del rango de los límites establecidos en los objetivos de calidad del agua de la Resolución 2016 del Área Metropolitana. En la gráfica 7 se puede observar que una vez se realiza el vertimiento se genera un decrecimiento en el parámetro analizado; sin embargo, también es perceptible que no hay un cambio significativo, a pesar de que el vertimiento presenta un valor elevado, y, por el contrario, tiende a la neutralidad.

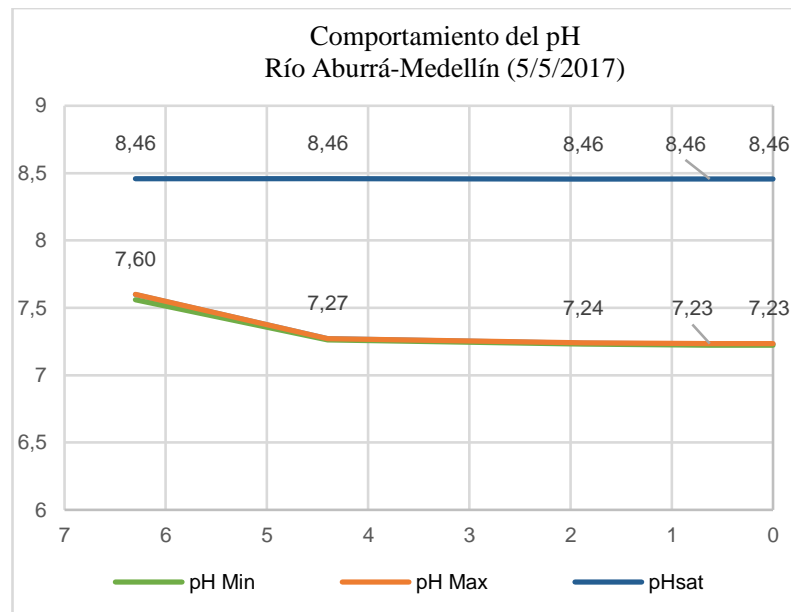

Gráfica 7. pH modelado para el tramo Niquía- Ancón Norte.

\section{Conclusiones}

Se realizó una evaluación de impacto ambiental relacionada con los vertimientos de una empresa de curtiembres usando el método Conesa. Para esta 
evaluación se incluyó información referente a las materias primas, los equipos y los insumos requeridos en los procesos asociados a los residuos líquidos generados en cada etapa. El resultado de la evaluación demuestra que el impacto calificado como significativo fue el uso y la modificación fisicoquímica del recurso hídrico, especialmente en las actividades de remojo, pelambre, desencalado y purga, piquelado, curtido, recurtido y teñido. Seguido de los impactos al suelo por la generación de residuos peligrosos provenientes del proceso y lodos resultantes del tratamiento de aguas residuales.

Se aplicó el modelo de simulación QUAL2K para predecir las condiciones de afectación del río. Al recibir las cargas contaminantes de los vertimientos de una empresa de curtiembres, fueron relacionados los datos de las estaciones de monitoreo Niquía y Ancón Norte ubicadas en la zona de influencia y los análisis de calidad del agua residual tratada. Los parámetros simulados fueron $\mathrm{pH}$, conductividad, $\mathrm{DBO}$ rápida y lenta, caudal y temperatura.

El pH del agua residual tratada se entrega a la fuente superficial con un valor de 8,5; según el modelo, no afecta el cauce, y con respecto al cumplimiento normativo, descrito en la Resolución 2016 del 2015, este se encuentra en el rango de cumplimiento de los objetivos de descontaminación.

El agua residual proveniente de la empresa excede los límites permisibles relacionados en la Resolución 0631 del 2015 en los parámetros DQO y DBO5, en un $203 \%$ y $150 \%$. En la simulación el resultado de la DBO rápida vertida, aumenta la concentración de $167 \mathrm{mg}$ O2/L hasta $185 \mathrm{mg} \mathrm{O} / \mathrm{L}$ en el tramo estudiado, y se aleja significativamente del objetivo de calidad de $50 \mathrm{mg}$ de $\mathrm{O} 2 / \mathrm{L}$.

El vertimiento de la curtiembre tiene una leve afectación de la calidad del agua referente a materia orgánica, la cual disuelve debido a la diferencia que existe entre el caudal medio de $18,98 \mathrm{~m} 3 / \mathrm{s}$ para el río y de $0,00147 \mathrm{~m} 3 / \mathrm{s}$ del vertimiento. El flujo promedio del vertimiento representa el $0,0077 \%$ del caudal total después de mezclarse con el cauce del río.

\section{Recomendación}

Se recomienda que las empresas del sector de las curtiembres ajusten sus tratamientos de agua residual con métodos biológicos, para cumplir con la Resolución 631 de 2015.

\section{Referencias}

[1] Centro Nacional de Producción Más Limpia. Diagnóstico y Estrategias proyecto gestión ambiental en el sector de curtiembres. 2004. Disponible en:

www.sirac.info/curtiembres/html/archivos/publicacione s/estratagiasdiagnostico.pdf

[2] CONESA, V. Guía metodológica para la evaluación de impacto ambiental. Ed. Mundi- Prensa $3^{\text {ra }}$ edición, Madrid 2003. ISBN 84-7114-647-9

[3] Caballero Escribano, C. Historia de los curtidos de las pieles. Alicante, España: Editorial Club Universitario 2013. ISBN 97-8849-948-749-6

[4] Lofrano, Giusy, et al. Chemical and biological treatment technologies for leather tannery chemicals and wastewaters: A review. En: SCIENCE OF THE TOTAL ENVIRONMENT. Septiembre, 2013. vol. 461-462, p. 265-281

[5] Ministerio de Ambiente, Vivienda y Desarrollo Territorial. Guía ambiental para la industria del curtido y preparado de cueros. Segunda Edición ed. Bogotá D.C.: Print Digital Ltda 2006. ISBN 9789589778548

[6] MCCANN, M. Capítulo 88 Cuero, pieles y calzado. En: enciclopedia de la OIT. D - INSHT (Instituto Nacional de Seguridad e Higiene en el Trabajo) 2012.

[7] Salas, G. Eliminación de sulfuros por oxidación en el tratamiento del agua residual de una curtiembre. Revista Peruana de Química e Ingeniería Química. Vol. 8 N. ${ }^{\circ} 1$ 2005. Inline ISSN. 1609-7599. PrintISSN: 1726-2208

[8] Carabias, J; Provencio, E y Cortinas, C. Manual de Procedimientos para el Manejo Adecuado de los Residuos de la Curtiduría. Primera Edición ed. México D.F.: Instituto Nacional de Ecología 1999.

[9] Cooman, K., et al. Tannery wastewater characterization and toxicity effects on Daphnia spp. En: Environmental Toxicology. vol. 18, No. 1, p. 45-51. 2003 https://doi.org/10.1002/tox.10094

[10] Aloy, M.; Folachier, A. y Vulliermet, B. Tannery and pollution. Lyon (France): Centre Technique du Cuir. 306 p. 1976.

[11] Thanikaivelan, P., Rao, J. R. y Nair, B. U. Development of leather processing method in narrow $\mathrm{pH}$ profile. Part 1: standardization of dehairing process, vol. 84 , no. 6, p. 276-284. 2000. 
Evaluación ambiental asociada a los vertimientos de aguas residuales generados por una empresa de curtiembres en la cuenca del río Aburrá

[12] Marsal, A., et al. Oxidizing unhairing process with hair recovery. Part I: experiments on the prior hair immunization. vol. 83, No. 47, p. 310-315. 1999.

[13] Arango, C. Proyecto Gestión Ambiental en la Industria de Curtiembre en Colombia. Colombia. p. 5. 2004

[14] CONESA, V. Guía metodológica para la evaluación de impacto ambiental. Ed. Mundi- Prensa 3ra edición, Madrid. ISBN 84-7114-647-9. 2003

[15] Área Metropolitana del Valle De Aburrá. Red de monitoreo ambiental en la cuenca hidrográfica del río Aburrá en jurisdicción del área. convenio 368 de 2014 adición I y II metropolitana. Septiembre 2016

http://www.metropol.gov.co/recursohidrico/Documents/ ParametrosCalidadRio/EstacionesAutomaticasMonitore oAguaSuperficial.pdf. Consultado el 12 de septiembre de 2017

[16] Universidad de Antioquia, et al. Red de monitoreo ambiental en la cuenca hidrográfica del río Aburrá Medellín en jurisdicción del Área Metropolitana FASE IV - Informe de calidad. Medellín. p. 1249-1255. 2014.

[17] Diaz, B. Modelación de la calidad del agua en el interceptor río Bogotá en los tramos Fucha-TunjueloCanoas. Universidad De Los Andes, 2004. p. 15-18

[18]O. Guarín-Villamizar, "Metodología para evaluación de la condición ambiental en microcuencas urbanas," Rev. UIS Ing., vol. 16, no. 2, pp. 141-150, 2017. Doi: https://doi.org/10.18273/revuin.v16n22017013. 